\title{
The ratio of urinary $\alpha 1$-microglobulin to microalbumin can be used as a diagnostic criterion for tubuloproteinuria
}

\author{
Hongwen Zhang, Fang Wang, Huijie Xiao, Yong Yao* \\ Department of Pediatric, Peking University First Hospital, Beijing, China.
}

\begin{abstract}
Summary Low-molecular-weight proteinuria is one of the characteristic clinical manifestations of renal tubular and interstitial diseases. Low-molecular-weight proteinuria is defined as excessive urinary loss of a1-microglobulin, $\beta 2$-microglobulin, or other low-molecular-weight plasma proteins. The current study examined the ratio of urinary a1-microglobulin to microalbumin in 24 Chinese pediatric patients with renal tubular and interstitial diseases, including 10 patients with Dent disease, 2 patients with Lowe syndrome, 6 patients with acute tubulointerstitial nephritis (ATIN), 4 patients with acute tubulointerstitial nephritis with uveitis syndrome (TINU), and 2 patients with nephronophthisis (NPHP). Patients with steroid-sensitive nephrotic syndrome, IgA nephropathy, Henoch-Schonlein purpura nephritis, or lupus nephritis served as control groups. In all of the patients with tubular and interstitial disease, urinary a1-microglobin increased 10-300-fold above the upper limit of the normal range, the ratio of urinary a1-microglobulin to microalbumin was greater than 1 , and the percentage of low-molecular-weight plasma proteins (LMWP) in urine was greater than $50 \%$ according to urine protein electrophoresis. There was close correlation between the ratio of urinary $\alpha 1$-microglobulin to microalbumin and the percentage of LMWP in urine according to urine protein electrophoresis $(r=\mathbf{0 . 7 9 7}, p=0.000)$. We suggested firstly that the ratio of urinary a1-microglobulin to microalbumin, greater than 1 , can be used as a diagnostic criterion for tubuloproteinuria.
\end{abstract}

Keywords: $\alpha 1$-microglobulinuria, microalbuminuria, tubuloproteinuria

\section{Introduction}

Low-molecular-weight proteinuria is one of the characteristic clinical manifestations of renal tubular and interstitial diseases, such as Dent disease, Lowe syndrome, acute tubulointerstitial nephritis (ATIN) without or with uveitis syndrome (TINU), Fanconi syndrome, and nephronophthisis (NPHP) $(1,2)$. Lowmolecular-weight proteinuria is usually detected with urine protein electrophoresis (SDS polyacrylamide gel electrophoresis), but this technique is complicated and time-consuming. Elevated levels of urinary $\beta 2-$ microglobulin and $\alpha 1$-microglobulin can also serve as markers of low-molecular-weight proteinuria, but there

Released online in J-STAGE as advance publication February 26, 2018.

*Address correspondence to:

Dr. Yong Yao, Department of Pediatric, Peking University First Hospital, No.1 Xi An Men Da Jie, Beijing 100034, China.

E-mail: yaoyong3238@126.com are no definitive levels of those plasma proteins in urine.

The current study examined the ratio of urinary $\alpha 1-$ microglobulin to microalbumin in several types of renal tubular and interstitial diseases that present in childhood in order to determine whether that ratio could be used as a diagnostic criterion for tubuloproteinuria.

\section{Subjects and Methods}

\subsection{Participants}

This study was approved by Peking University First Hospital (No. 2014-826) and followed the guidelines of the 2000 Declaration of Helsinki and the 2008 Declaration of Istanbul. Consent was obtained from all patients and their families.

Data were collected from 24 Chinese pediatric patients with renal tubular and interstitial diseases, including 10 patients with Dent disease, 2 patients with Lowe syndrome, 6 patients with ATIN, 4 patients with 
TINU, and 2 patients with NPHP. Patients were seen from January 01, 2014 to December 31, 2016 and were analyzed retrospectively. All patients presented with proteinuria. Patients with steroid-sensitive nephrotic syndrome (NS), IgA nephropathy, Henoch-Schonlein purpura nephritis, or lupus nephritis were also included as control groups. These patients were seen during the same period, and each group consisted of 20 patients. Data were collected upon diagnosis.

The clinical diagnosis of Dent's disease is based on meeting all three of the following criteria: $i$ ) lowmolecular-weight proteinuria (elevated urinary excretion of $\alpha 1$-microglobulin at least 100-fold greater than the upper limit of the normal range, or greater than $50 \%$ low-molecular-weight plasma proteins (LMWP) in urine according to urine protein electrophoresis); $i$ ) hypercalciuria $(>0.1 \mathrm{mmol} / \mathrm{kg}$ according to 24 -hour urine collection or $>0.21 \mathrm{mg} / \mathrm{mg}$ calcium to creatinine ratio according to a spot sample); and iii) at least one of the following: nephrocalcinosis, kidney stones, hematuria, hypophosphatemia, or renal insufficiency.

The identification of a mutation in either CLCN5 or OCRL1 confirms the diagnosis $(3,4)$. The clinical diagnosis of Lowe syndrome is based on meeting three of the following criteria: congenital bilateral cataracts, renal Fanconi syndrome, hypotonia and neonatal areflexia, motor and mental developmental delays, and facial dysmorphisms. The identification of a mutation in OCRL1 confirms the diagnosis $(5,6)$.

The clinical diagnosis of ATIN without or with uveitis is based on the clinical course and laboratory examinations including urinalysis and a renal function test. A percutaneous renal biopsy reveals diffuse interstitial nephritis with infiltration of neutrophil and eosinophil granulocytes and numerous lymphocytes and plasma cells. No granuloma is found. Typical bilateral anterior uveitis concurrent with or preceding or following the onset of renal dysfunction corroborates the diagnosis of $\operatorname{TINU}(7,8)$.

The clinical diagnosis of NPHP is based on multiple organ involvement that includes at least abnormal renal and liver function or cysts, and urinalysis may reveal a renal concentration defect. The identification of a mutation in NPHPs confirms the diagnosis $(9,10)$.

\subsection{Clinic and laboratory examinations}

Results of all examinations, such as a quantitative test of 24-h urinary protein, were examined. The presence of $\alpha 1$-microglobinuria and microalbuminuria was also determined. Results of urine protein electrophoresis were examined.

\subsection{Statistical analysis}

Statistical analysis was performed with SSPS 12.0 software. Every index was measured three times and summarized as the mean $\pm \mathrm{SD}$, and the relationship between the ratio of urinary $\alpha 1$-microglobulin to microalbumin and the percentage of LMWP in urine according to urine protein electrophoresis was analyzed using Pearson's correlation coefficient. An independentsamples test was used to examine the mean ratio of urinary $\alpha 1$-microglobulin to microalbumin and the mean percentage of LMWP in urine in patients with renal tubular and interstitial disease and the control groups. $P$ values less than 0.05 were considered statistically significant.

\section{Results and Discussion}

All 24 patients presented with proteinuria. Patients with Dent disease or Lowe syndrome had proteinuria in the nephrotic range while patients with ATIN, TINU, or NPHP had proteinuria that did not fall in the nephrotic range. Urinary $\alpha 1$-microglobin and microalbumin both increased significantly in all patients. This was especially true for $\alpha 1$-microglobin, which increased 10-300-fold above the upper limit of the normal range. In all patients, the percentage of LMWP in urine was greater than $50 \%$ according to urine protein electrophoresis, and the ratio of urinary $\alpha 1$-microglobulin to microalbumin was greater than 1. Urinary $\alpha 1$-microglobin and microalbumin and the percentage of LMWP in urine were closely correlated $(r$ $=0.797, p=0.000$ ), as shown in Table 1 .

In contrast, urinary microalbumin increased substantially but $\alpha 1$-microglobin was almost normal in all four control groups. There were almost no LMWP in urine according to urine protein electrophoresis, and the ratio of urinary $\alpha 1$-microglobulin to microalbumin was near zero. The level of $\alpha 1$-microglobin, the ratio of urinary $\alpha 1$-microglobulin to microalbumin, and the percentage of LMWP in urine according to urine protein electrophoresis were much higher in patients with renal tubular and interstitial diseases than those in the four control groups $(p<0.01)$, while the level of microalbumin was much lower in patients with renal tubular and interstitial diseases than that in the four control groups $(p<0.01)$, as shown in Table 2 .

Low-molecular-weight proteinuria is one of the major clinical manifestations of renal tubular and interstitial diseases. Low-molecular-weight proteinuria is defined as excessive urinary loss of $\alpha 1$-microglobulin, $\beta 2$-microglobulin, or other low-molecular-weight plasma proteins. The current study examined the ratio of urinary $\alpha 1$-microglobulin to microalbumin in several types of renal tubular and interstitial diseases that present in childhood, including Dent disease, Lowe syndrome, acute tubulointerstitial nephritis, and nephronophthisis, in order to find an easy, simple, and quick diagnostic criterion for tubuloproteinuria.

Dent disease is an $\mathrm{X}$-linked recessive renal tubulopathy, and low-molecular-weight proteinuria is its 
Table 1. Clinical data on 24 patients with renal tubular and interstitial diseases

\begin{tabular}{llcccccc}
\hline Patient & Diagnosis & Age at diagnosis & UPE $(\mathrm{mg} / \mathrm{kg} / 24 \mathrm{~h})$ & $\alpha 1-\mathrm{MG}(\mathrm{mg} / \mathrm{L})$ & MA $(\mathrm{mg} / \mathrm{L})$ & $\alpha 1-\mathrm{MG} / \mathrm{MA}$ & LMWP $(\%)$ \\
\hline No. 1 & DS* & $9.8 \mathrm{y}$ & $64 \pm 18$ & $402 \pm 113$ & $285 \pm 89$ & $1.51 \pm 0.12$ & $61.3 \pm 9.3$ \\
No. 2 & DS & $5.8 \mathrm{y}$ & $58 \pm 17$ & $349 \pm 125$ & $301 \pm 81$ & $1.33 \pm 0.14$ & $53.1 \pm 7.5$ \\
No. 3 & DS & $5.2 \mathrm{y}$ & $55 \pm 15$ & $305 \pm 108$ & $257 \pm 76$ & $1.31 \pm 0.11$ & $53.5 \pm 8.4$ \\
No. 4 & DS & $4.5 \mathrm{y}$ & $51 \pm 16$ & $346 \pm 101$ & $273 \pm 86$ & $1.35 \pm 0.12$ & $51.3 \pm 8.1$ \\
No. 5 & DS & $5.7 \mathrm{y}$ & $53 \pm 14$ & $375 \pm 134$ & $268 \pm 79$ & $1.40 \pm 0.10$ & $56.5 \pm 7.2$ \\
No. 6 & DS & $5.8 \mathrm{y}$ & $53 \pm 13$ & $382 \pm 120$ & $258 \pm 82$ & $1.51 \pm 0.16$ & $59.5 \pm 7.6$ \\
No. 7 & DS & $3.5 \mathrm{y}$ & $62 \pm 19$ & $353 \pm 131$ & $249 \pm 73$ & $1.33 \pm 0.15$ & $52.8 \pm 9.0$ \\
No. 8 & DS & $1.5 \mathrm{y}$ & $50 \pm 17$ & $258 \pm 102$ & $197 \pm 68$ & $1.43 \pm 0.13$ & $56.3 \pm 6.5$ \\
No.9 & DS & $5.1 \mathrm{y}$ & $56 \pm 11$ & $341 \pm 116$ & $238 \pm 62$ & $1.42 \pm 0.12$ & $56.1 \pm 7.5$ \\
No. 10 & DS & $5.2 \mathrm{y}$ & $57 \pm 10$ & $355 \pm 124$ & $248 \pm 61$ & $1.44 \pm 0.13$ & $55.9 \pm 6.3$ \\
No. 11 & LS* & $4.6 \mathrm{y}$ & $49 \pm 12$ & $315 \pm 106$ & $216 \pm 57$ & $1.46 \pm 0.11$ & $54.8 \pm 7.2$ \\
No. 12 & LS & $4.9 \mathrm{y}$ & $45 \pm 11$ & $302 \pm 108$ & $237 \pm 62$ & $1.27 \pm 0.10$ & $53.2 \pm 6.6$ \\
No. 13 & ATIN* & $7.5 \mathrm{y}$ & $22 \pm 5$ & $136 \pm 14$ & $102 \pm 13$ & $1.33 \pm 0.14$ & $54.7 \pm 7.6$ \\
No. 14 & ATIN & $8.2 \mathrm{y}$ & $17 \pm 4$ & $121 \pm 12$ & $98 \pm 11$ & $1.24 \pm 0.11$ & $51.6 \pm 7.2$ \\
No. 15 & ATIN & $9.4 \mathrm{y}$ & $25 \pm 6$ & $135 \pm 14$ & $112 \pm 16$ & $1.21 \pm 0.13$ & $50.3 \pm 6.8$ \\
No. 16 & ATIN & $10.8 \mathrm{y}$ & $19 \pm 5$ & $124 \pm 16$ & $89 \pm 12$ & $1.40 \pm 0.16$ & $54.8 \pm 6.5$ \\
No. 17 & ATIN & $12.5 \mathrm{y}$ & $23 \pm 7$ & $130 \pm 15$ & $115 \pm 14$ & $1.13 \pm 0.14$ & $52.5 \pm 6.9$ \\
No. 18 & ATIN & $9.8 \mathrm{y}$ & $25 \pm 5$ & $138 \pm 17$ & $120 \pm 13$ & $1.15 \pm 0.13$ & $51.3 \pm 7.2$ \\
No. 19 & TINU* & $12.4 \mathrm{y}$ & $27 \pm 8$ & $141 \pm 18$ & $124 \pm 15$ & $1.17 \pm 0.15$ & $52.7 \pm 6.8$ \\
No. 20 & TINU & $10.8 \mathrm{y}$ & $19 \pm 4$ & $118 \pm 16$ & $88 \pm 12$ & $1.34 \pm 0.15$ & $55.7 \pm 7.1$ \\
No. 21 & TINU & $11.3 \mathrm{y}$ & $12 \pm 3$ & $98 \pm 10$ & $81 \pm 10$ & $1.21 \pm 0.11$ & $53.1 \pm 6.9$ \\
No. 22 & TINU & $13.6 \mathrm{y}$ & $18 \pm 5$ & $105 \pm 12$ & $94 \pm 11$ & $1.12 \pm 0.14$ & $52.7 \pm 6.6$ \\
No. 23 & NPHP* & $3.8 \mathrm{y}$ & $28 \pm 5$ & $109 \pm 16$ & $87 \pm 15$ & $1.25 \pm 0.10$ & $53.5 \pm 7.1$ \\
No. 24 & NPHP & $9.6 \mathrm{y}$ & $23 \pm 4$ & $98 \pm 11$ & $85 \pm 12$ & $1.15 \pm 0.12$ & $52.4 \pm 7.3$ \\
\end{tabular}

*DS: Dent disease; LS: Lowe syndrome; ATIN: Acute tubulointerstitial nephritis; TINU: Tubulointerstitial nephritis with uveitis; NPHP: Nephronophthisis.

Table 2. Levels of $\alpha 1-M G$, MA, and $\alpha 1-M G / M A$ and the percentage of LMWP in different groups

\begin{tabular}{|c|c|c|c|c|c|}
\hline Groups & Age (years) & $\alpha 1-\mathrm{MG}(\mathrm{mg} / \mathrm{L})$ & $\mathrm{MA}(\mathrm{mg} / \mathrm{L})$ & $\alpha 1-\mathrm{MG} / \mathrm{MA}$ & LMWP (\%) \\
\hline Renal tubular and interstitial diseases & $7.6 \pm 3.4$ & $231 \pm 78^{\mathbf{4}}$ & $176 \pm 43^{\wedge}$ & $1.31 \pm 0.14^{\mathbf{\Lambda}}$ & $54.2 \pm 7.3^{\mathbf{\Lambda}}$ \\
\hline Nephrotic syndrome & $6.8 \pm 3.1$ & $0 \pm 0$ & $3015 \pm 483$ & $0 \pm 0$ & $0 \pm 0$ \\
\hline IgA nephropathy & $7.8 \pm 1.7$ & $8 \pm 2$ & $1853 \pm 665$ & $0 \pm 0$ & $3.5 \pm 1.6$ \\
\hline Henoch-Schonlein Purpura nephritis & $7.9 \pm 2.3$ & $6 \pm 2$ & $1873 \pm 587$ & $0 \pm 0$ & $3.2 \pm 1.5$ \\
\hline Lupus nephritis & $8.5 \pm 1.6$ & $9 \pm 3$ & $2981 \pm 621$ & $0 \pm 0$ & $6.4 \pm 3.0$ \\
\hline
\end{tabular}

: $p<0.01$ compared to other groups.

most constant feature. It mainly affects male children, and female carriers are generally asymptomatic $(11,12)$. The level of proteinuria often reaches the nephrotic range $(13,14)$. This hampers the diagnosis of Dent disease and explains why it is often treated as NS (15-17). The current results indicated that urinary $\alpha 1$-microglobin increased markedly (200-300-fold above the upper limit of normal, $0-12 \mathrm{mg} / \mathrm{L}$ ) in all 10 patients with Dent disease, and the ratio of urinary $\alpha 1$ microglobulin to microalbumin was greater than 1 . This suggests that the ratio of urinary $\alpha 1$-microglobulin to microalbumin could be used to measure LMWP in urine in patients with Dent disease.

Lowe syndrome is also an $\mathrm{X}$-linked recessive renal tubulopathy that involves the eyes, central nervous system, and kidneys. Renal disease is primarily characterized by renal Fanconi syndrome, including lowmolecular-weight proteinuria, proximal renal tubular acidosis, renal phosphate wasting, hypercalciuria, aminoaciduria, and hypokaliemia $(5,6)$. The current results indicated that urinary $\alpha 1$-microglobin also increased markedly (about 300-fold above the upper limit of the normal range) in the 2 patients with Lowe syndrome, and the ratio of urinary $\alpha 1$-microglobulin to microalbumin was greater than 1 . This suggests that the ratio of urinary $\alpha 1$-microglobulin to microalbumin could be used to measure LMWP in urine in patients with Lowe syndrome.

Tubulointerstitial nephritis (TIN) is characterized histologically by inflammation of and damage to tubulointerstitial structures, with relative sparing of glomerular and vascular elements. The clinical manifestations of TIN vary. The severity of renal impairment ranges from asymptomatic urinary abnormalities to mild azotemia, and even to nonoliguric and oliguric acute renal failure (ARF) $(18,19)$. The nonspecific nature of the clinical findings in TIN highlights the need to perform a renal biopsy to make a definitive diagnosis in questionable cases. Systemic manifestations of hypersensitivity, such as a fever, rash, and arthralgia, vary. However, mild to moderate proteinuria (less than $1 \mathrm{~g}$ /day, mainly low-molecular- 
weight proteinuria) is found in most patients with TIN. When tubulointerstitial nephritis is combined with uveitis, the condition is known as tubulointerstitial nephritis and uveitis (TINU) syndrome. This condition is mainly seen in children with a favorable renal prognosis (2). The diagnosis of TIN or TINU is difficult because of variable and nonspecific clinical manifestations. Studies have reported that urinary $\alpha 1$-microglobulin and $\beta 2$-microglobulin excretion increase in patients with TINU $(20,21)$. The current results indicated that urinary a1-microglobin increased significantly (about 100fold above the upper limit of the normal range) in the 6 patients with ATIN and in the 4 patients with TINU, and the ratio of urinary $\alpha 1$-microglobulin to microalbumin was greater than 1 . This suggests that the ratio of urinary a1-microglobulin to microalbumin could be used to measure LMWP in urine in patients with TIN or TINU.

Nephronophthisis (NPHP) includes a group of rare autosomal-recessive cystic kidney diseases, characterized by a broad genetic and clinical heterogeneity and accounting for the majority of genetic causes of endstage renal disease (ESRD) during childhood. NPHP is associated with extra renal manifestations in 10$15 \%$ of patients $(22,23)$. The diagnosis of NPHP is difficult because of its genetic and clinical complexity (24). However, the kidneys and liver are involved in most cases, and a low urine specific gravity and lowmolecular-weight proteinuria are commonly seen in urinalysis (25). The current results indicated that urinary a1-microglobin increased close to 100 -fold above the upper limit of the normal range in 2 patients with NPHP, and the ratio of urinary $\alpha 1$-microglobulin to microalbumin was greater than 1 . This suggests that the ratio of urinary $\alpha 1$-microglobulin to microalbumin could be used to measure LMWP in urine in patients with NPHP. However, albuminuria in the nephrotic range is also seen in a few patients with NPHP (26), and LMWP in urine is not always specific to NPHP.

NS, IgA nephropathy, Henoch-Schonlein purpura nephritis, and lupus nephritis are the most common glomerular diseases that present during childhood. Proteinuria caused by these glomerular diseases mainly involves albumin. Upon diagnosis, urinary microalbumin increased markedly but $\alpha 1$-microglobin was almost normal in all 4 control groups, there were almost no LMWP in urine according to urine protein electrophoresis, and the ratio of urinary $\alpha 1$-microglobulin to microalbumin was near zero. The ratio of urinary $\alpha 1$ microglobulin to microalbumin differed substantially from that in patients with renal tubular and interstitial diseases.

In conclusion, this study is the first to suggest that a ratio of urinary $\alpha 1$-microglobulin to microalbumin greater than 1 can be used as a diagnostic criterion for tubuloproteinuria. However, this study has several limitations. The sample size was small, few types of renal tubular and interstitial diseases were examined, and only pediatric patients were included. Multicenter and larger-scale studies are needed to verify the current results.

\section{References}

1. Sekine T, Komoda F, Miura K, Takita J, Shimadzu M, Matsuyama T, Ashida A, Igarashi T. Japanese Dent disease has a wider clinical spectrum than Dent disease in Europe/ USA: Genetic and clinical studies of 86 unrelated patients with low-molecular-weight proteinuria. Nephrol Dial Transplant. 2014; 29:376-384.

2. Sinnamon KT, Courtney AE, Harron C, O'Rourke DM, Mullan RN. Tubulointerstitial nephritis and uveitis (TINU) syndrome: Epidemiology, diagnosis and management. NDT Plus. 2008; 1:112-116.

3. Hoopes RR Jr, Raja KM, Koich A, Hueber P, Reid R, Knohl SJ, Scheinman SJ. Evidence for genetic heterogeneity in Dent's disease. Kidney Int. 2004; 65:1615-1620

4. Grand T, L'Hoste S, Mordasini D, Defontaine N, Keck M, Pennaforte T, Genete M, Laghmani K, Teulon J, Lourdel $\mathrm{S}$. Heterogeneity in the processing of CLCN5 mutants related to Dent disease. Hum Mutat. 2011; 32:476-483.

5. Cho HY, Lee BH, Choi HJ, Ha IS, Choi Y, Cheong HI. Renal manifestations of Dent disease and Lowe syndrome. Pediatr Nephrol. 2008; 23:243-249.

6. Bockenhauer D, Bokenkamp A, van't Hoff W, Levtchenko E, Kist-van Holthe JE, Tasic V, Ludwig M. Renal phenotype in Lowe Syndrome: A selective proximal tubular dysfunction. Clin J Am Soc Nephrol. 2008; 3:1430-1436.

7. Mandeville JT, Levinson RD, Holland GN. The tubulointerstitial nephritis and uveitis syndrome. Surv Ophthalmol. 2001; 46:195-208.

8. Saarela V, Nuutinen M, Ala-Houhala M, Arikoski P, Ronnholm K, Jahnukainen T. Tubulointerstitial nephritis and uveitis syndrome in children: A prospective multicenter study. Ophthalmology. 2013; 120:1476-1481.

9. Salomon R, Saunier S, Niaudet P. Nephronophthisis. Pediatr Nephrol. 2009; 24:2333-2344.

10. Simms RJ, Eley L, Sayer JA. Nephronophthisis. Eur J Hum Genet. 2009; 17:406-416.

11. Claverie-Martin F, Ramos-Trujillo E, Garcia-Nieto V. Dent's disease: Clinical features and molecular basis. Pediatr Nephrol. 26: 693-704.

12. Devuyst O. Dent's disease: Chloride-proton exchange controls proximal tubule endocytosis. Nephrol Dial Transplant. 2010; 25:3832-3835.

13. Li P, Huang JP. Phenotype and genotype of Dent's disease in three Chinese boys. Nephrology (Carlton). 2009; 14:139-142.

14. Jian S, Wei M, He YY, Wang W, Kang YL, Sun ZX. Clinical and genetic analysis of Dent disease in 4 Chinese children. Zhongguo Dang Dai Er Ke Za Zhi. 2015; 17:1261-1266. (in Chinese)

15. Frishberg Y, Dinour D, Belostotsky R, Becker-Cohen R, Rinat C, Feinstein S, Navon-Elkan P, Ben-Shalom E. Dent's disease manifesting as focal glomerulosclerosis: Is it the tip of the iceberg? Pediatr Nephrol. 2009; 24:23692373.

16. Szczepanska M, Zaniew M, Recker F, Mizerska-Wasiak M, Zaluska-Lesniewska I, Kilis-Pstrusinska K, Adamczyk 
P, Zawadzki J, Pawlaczyk K, Ludwig M, Sikora P. Dent disease in children: Diagnostic and therapeutic considerations. Clin Nephrol. 2015; 84:222-230.

17. Cramer MT, Charlton JR, Fogo AB, Fathallah-Shaykh SA, Askenazi DJ, Guay-Woodford LM. Expanding the phenotype of proteinuria in Dent disease. A case series. Pediatr Nephrol. 2014; 29:2051-2054.

18. Greising J, Trachtman H, Gauthier B, Valderrama E. Acute interstitial nephritis in adolescents and young adults. Child Nephrol Urol. 1990; 10:189-195.

19. Jones CL, Eddy AA. Tubulointerstitial nephritis. Pediatr Nephrol. 1992; 6:572-586.

20. Li C, Su T, Chu R, Li X, Yang L. Tubulointerstitial nephritis with uveitis in Chinese adults. Clin J Am Soc Nephrol. 2014; 9:21-28.

21. Hettinga YM, Scheerlinck LM, Lilien MR, Rothova $\mathrm{A}$, de Boer JH. The value of measuring urinary $\beta 2$ microglobulin and serum creatinine for detecting tubulointerstitial nephritis and uveitis syndrome in young patients with uveitis. JAMA Ophthalmol. 2015; 133:140-
145.

22. Wolf MT, Hildebrandt F. Nephronophthisis. Pediatr Nephrol. 2011; 26:181-194.

23. Benzing T, Schermer B. Clinical spectrum and pathogenesis of nephronophthisis. Curr Opin Nephrol Hypertens. 2012; 21:272-278.

24. O'Toole JF, Otto EA, Hoefele J, Helou J, Hildebrandt F. Mutational analysis in 119 families with nephronophthisis. Pediatr Nephrol. 2007; 22:366-370.

25. Sugimoto K, Miyazawa T, Enya T, Nishi H, Miyazaki K, Okada M, Takemura T. Clinical and genetic characteristics of Japanese nephronophthisis patients. Clin Exp Nephrol. 2015; 20:637-649.

26. Zhang H, Su B, Liu X, Xiao H, Ding J, Yao Y. Mutations in TTC21B cause different phenotypes in two childhood cases in China. Nephrology (Carlton). 2017. doi: 10.1111/ nep. 13008 .

(Received December 7, 2017; Revised January 28, 2018; Accepted February 1, 2018) 